\section{Emerging health concerns about pesticide residues}

\author{
William Pease
}

F or more than 30 years, most public ticide residues in food has focused on whether life-long exposure to some chemicals in the diet could increase the incidence of cancer. The Delaney Clause, a 1958 law banning the use of animal carcinogens as food additives, remains at the center of the current Congressional debate over pesticide reform. There is growing interest, however, in examining whether pesticide exposures could have other adverse effects on human health. Public concern about diseases other than cancer has been stimulated by the results obtained from more thorough toxicity testing of pesticides. As scientific understanding of the full range of pesticide toxicity has improved, a new question is being asked in policy debates: Can exposure to pesticide residues in our diet harm our reproductive, neurological or immune systems?

Assessing these potential risks involves many of the same scientific uncertainties that plague current efforts to estimate cancer risks. It is clear from the results of toxicological studies on laboratory animals that many pesticides in widespread use have the capacity to damage important physiological systems. There is also epidemiological evidence that workers with relatively high levels of pesticide exposure can suffer from a variety of acute and chronic health effects.

Some pesticides are reproductive toxicants, capable of damaging the human reproductive system. The normal sperm count of workers formulating DBCP (dibromochloropropane), for example, was lowered substantially because this nematocide is toxic to critical germinal cells in the male testes. Highly exposed workers became infertile and were unable to father children. Other pesticides (for example, organochlorine insecticides like endosulfan) mimic the activity of hu- man hormones and may affect female reproductive capacity. Toxicological evidence indicates that many pesticides can also cause birth defects: Of some 200 pesticides tested to date, nearly half are reported to induce birth defects in experimental animals.

Some pesticides can damage the immune system, triggering hypersusceptiblity to chemical exposures or hindering an organism's ability to successfully fight off infections. Occupational exposures to captan, for example, can induce contact hypersensitivity, a type of dermatitis. Toxicological studies have demonstrated that other pesticides can damage the immune system. Pesticides such as carbofuran and methyl parathion, for example, reduce an experimental animal's capacity to resist bacterial infections.

Entire classes of pesticides, like the organophosphate and carbamate insecticides, target enzymes that are essential to the functioning of a healthy nervous system. Farmworker exposures to mevinphos and methomyl, for example, have resulted in several mass poisoning incidents. Workers experience both physical and neurological symptoms, including nausea, breathing difficulties, irritability and confusion. Epidemiological studies indicate there can also be long-term damage (such as memory disturbances and deficits in intelligence and motor functioning) as a result of repeated acute exposures to neurotoxic pesticides.

Whether these types of adverse effects are occurring in humans as a result of dietary exposures to pesticide residues is largely unknown. Such endpoints often involve nonspecific symptoms. It is also difficult to characterize the pesticide exposures people experience. There is a complex mixture of many different pesticides in normal diets, as well as a number of other potential routes of exposure (for example, home consumer product

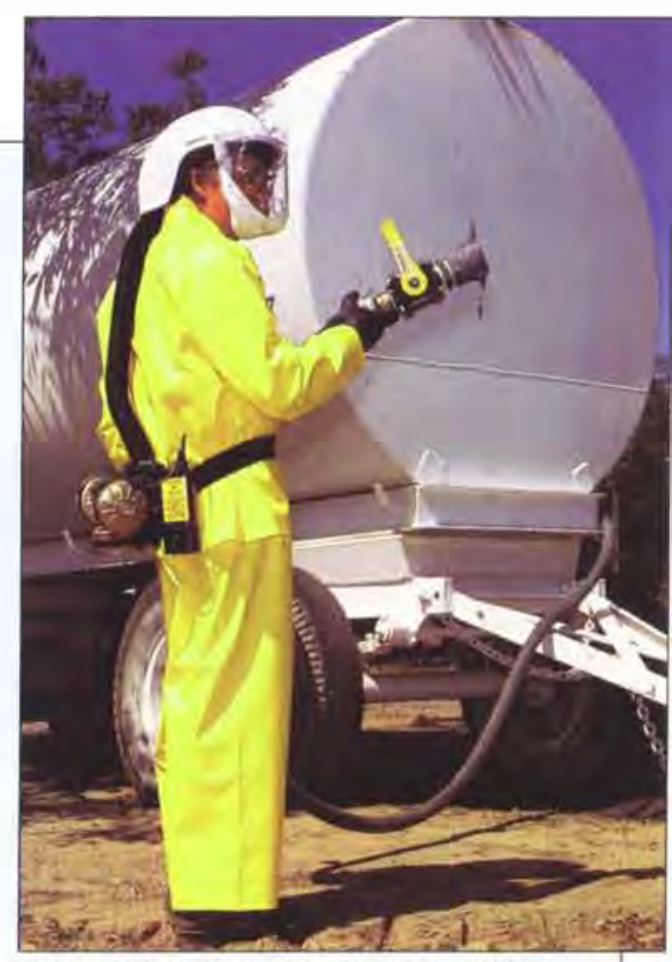

This pesticide applicator is outfitted with a respirator and protective clothing. Occupational exposures to pesticides are far greater than those from food consumption.

use). This has made it virtually impossible to conduct epidemiological studies of the noncancer effects of lowlevel dietary exposures.

Current scientific efforts compare estimated dietary intakes with pesticide dose levels known to cause adverse effects. The recent NAS report on pesticides in children's diets, for example, examined whether the cumulative intake of different pesticides that inhibit cholinesterase could have adverse neurological consequences. The report found that while the vast majority of children do not consume sufficient quantities of pesticide residues to raise any health concerns, about $1 \%$ of children on any given day are receiving pesticide doses that exceed EPA's acceptable exposure level. This finding has not resolved whether serious noncancer effects can be associated with food residues. Rather, it represents a new phase in the debate over food safety, as regulators begin to focus on reducing cumulative effects potentially associated with the variety of pesticide residues in the food supply.

W. Pease is Toxicologist, Environmental Health Sciences, School of Public Health, UC Berkeley. 